\title{
A subwavelength micropolarizer in a gold film for visible light
}

\author{
Victor V. Kotlyar , ${ }^{1,2}$ Sergey S. Stafeev, $1,2,{ }^{*}$ Maria V. Kotlyar , 2 \\ Anton G. NALIMOV, 1,2 LIAM O'FAOLAin ${ }^{3}$ \\ 1 Image Processing Systems Institute of the RAS, 151 Molodogvardeyskaya st., 443001, Samara, Russia \\ ${ }^{2}$ Samara State Aerospace University, 34 Moskovskoe shosse, 443086, Samara, Russia \\ ${ }^{3}$ School of Physics \& Astronomy, University of St. Andrews, North Haugh, St. Andrews, KY16 9SS, Scotland, UK \\ *Corresponding author: sergey.stafeev@gmail.com
}

Received XX Month XXXX; revised XX Month, XXXX; accepted XX Month XXXX; posted XX Month XXXX (Doc. ID XXXXX); published XX Month XXXX

\begin{abstract}
We have designed and fabricated a $100 \times 100-\mu \mathrm{m}$ four-sector binary subwavelength reflecting polarization microconverter in a gold film. Using the FDTD-aided numerical simulation and experiments, the micropolarizer was shown to convert an incident linearly polarized Gaussian beam of wavelength $532 \mathrm{~nm}$ into an azimuthally polarized beam. Conditions for generating on-axis regions of non-zero intensity when using propagating optical vortices with different initial polarization were deduced. By putting a spiral phase plate into an azimuthally polarized beam the intensity pattern was shown to change from diffraction rings to a central peak. (C) 2015 Optical Society of America
\end{abstract}

OCIS codes: (050.1380) Binary optics; (050.4865) Optical vortices; (050.6624) Subwavelength structures; (220.4000) Microstructure fabrication; (230.5440) Polarization-selective devices; (260.5430) Polarization.

http://dx.doi.org/10.1364/AO.99.099999

\section{Introduction}

Recent years have seen the publication of a large number of articles handling techniques for generating cylindrical vector beams. A one way to obtain cylindrical vector beams is through the use of subwavelength diffraction gratings. By properly choosing the feature height in such a grating, it may serve as a half- (or quarter-) wave plate, meanwhile the groove direction defines the spatial orientation of the plate.

For the first time, the use of the subwavelength gratings as polarization converters was reported in [1]. As the first publications to report on the fabrication of the gratings, we may denote Refs. [2, 3], where a circularly polarized $10.6-\mu \mathrm{m}$ light beam was converted into an azimuthally polarized beam. Generation of a radially polarized light beam for a subwavelength grating operating at a 1064-nm wavelength was discussed in [4]. A technique for fabricating a polarization converter in silicon for wavelengths ranging from 1030 to $1060 \mathrm{~nm}$ was proposed in [5].

It is worth noting that the majority of papers concerned with the use of subwavelength gratings for polarization conversion analyze the IR range of wavelengths because the fabrication of diffraction gratings for the visible light is a technologically challenging task. We have only managed to find one paper [6] in which a subwavelength annular diffraction grating fabricated in aluminum performed the conversion of a circularly polarized beam into a radially polarized one for a visible wavelength of $633 \mathrm{~nm}$. Note, however, that such a grating is unable to form an azimuthally polarized beam. Earlier, we discussed a four-zone grating polarizer intended to form a radially polarized beam [7]. In a follow-up study, we used the resulting radially polarized beam to form a subwavelength focal spot of area $(0.35 \times 0.38) \lambda$ by means of a Fresnel zone plate [8]. The radially polarized light has been primarily used for the sharp focusing of light. In recent years, the interest of researchers has been attracted to the analysis of optical vortices with radial and azimuthal polarization.

To the best of our knowledge, the first work to study the focusing of non-uniformly polarized optical vortices was reported in [9]. In particular, using the Richards-Wolf integrals, the researchers have shown that a radially or azimuthally polarized optical vortex is able to form a focal spot with central intensity peak only for the topological charge equal to $n=1$. Focusing radially and azimuthally polarized optical vortices with the topological charges $n=1$ and $n=2$ in a birefringent crystal was numerically studied in [10]. The vortex with $n=1$ was shown to generate an intensity peak in the focus of a lens with NA=0.9, whereas the vortex with the topological charge $n=2$ generated an annular intensity pattern. The propagation of an optical vortex initially characterized by radial polarization was analyzed in [11]. The effect of a spiral plate introduced into an azimuthally polarized laser beam to obtain a tighter focal spot was studied in [12]. Such a beam was shown to generate a focal spot of the area $0.147 \lambda^{2}$, which is $13.5 \%$ smaller when compared to a similar focal spot from a radially polarized beam $\left(0.17 \lambda^{2}\right)$. In [13], an azimuthally polarized beam was transmitted through concentric annular filters, with the 
rings acting as spiral plates arranged so that to ensure a phase shift of $\pi$ between the adjacent rings. The beam was focused with a wideaperture lens $(\mathrm{NA}=0.95)$ within a magneto-optical material, forming a needle-shaped focus of diameter FWHM $=0.38 \lambda$ and depth 7.48 . In a similar way, a radially polarized optical vortex was reported in [14] to

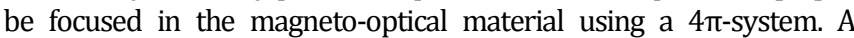
radially polarized optical vortex transmitted through an annular mask and focused by a $4 \pi$-system has been reported to generate foci chains over $30 \lambda$ long [15]. It should be noted that in the above-mentioned papers, the focusing of radially and azimuthally polarized beams was studied numerically, mainly with the use of the Richards-Wolf formulae. The only experimental research we have managed to discover [16] reported on the focusing of an azimuthally polarized optical vortex by means of a lens with NA $=1.4$. The resulting focal spot had an area of $0.089 \lambda^{2}$, which is $31 \%$ smaller than a focal spot from a circularly polarized beam.

In this work, we fabricated and studied the performance of a $100 \times 100$ $\mu \mathrm{m}$ four-sector binary subwavelength grating polarizer in a golden film. It was experimentally demonstrated that a linearly polarized 532 $\mathrm{nm}$ Gaussian beam reflected at the polarizer was converted to an azimuthally polarized beam. Putting a spiral phase plate (SPP) with the topological charge $n=1$ into the azimuthally polarized beam from the micropolarizer was experimentally shown to enable the conversion of the annular intensity pattern into a central intensity peak.

\section{Numerical simulation of the micropolarizer performance}

The proposed micropolarizer consists of four sectors (Fig. 1a): in the two sectors on the right, the microrelief features have a $0.46-\mu \mathrm{m}$ period and make angles $70^{\circ}$ and $-70^{\circ}$ with the $y$-axis (vertical), whereas in the sectors on the left, the features have a $0.4-\mu \mathrm{m}$ period and make with the $y$-axis angles $40^{\circ}$ and $-40^{\circ}$. The micropolarizer has a size of $100 \times 100 \mu \mathrm{m}$ and the microrelief height of $110 \mathrm{~nm}$.

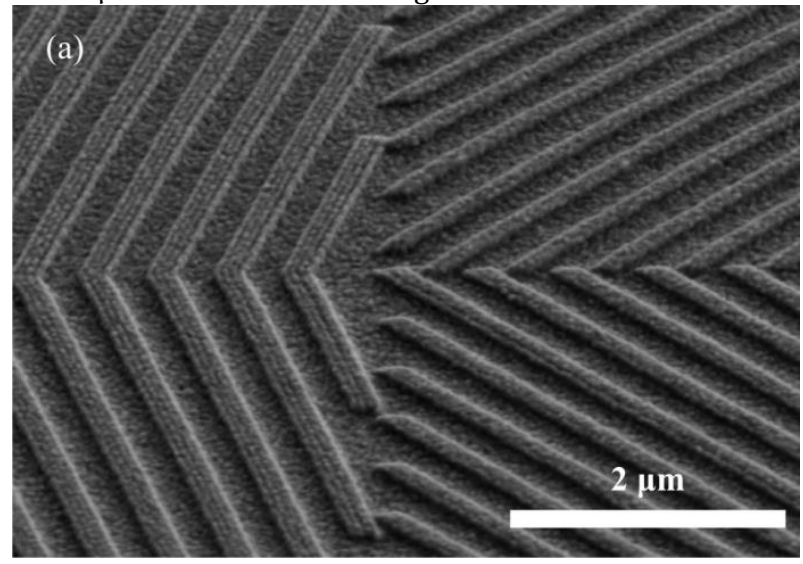

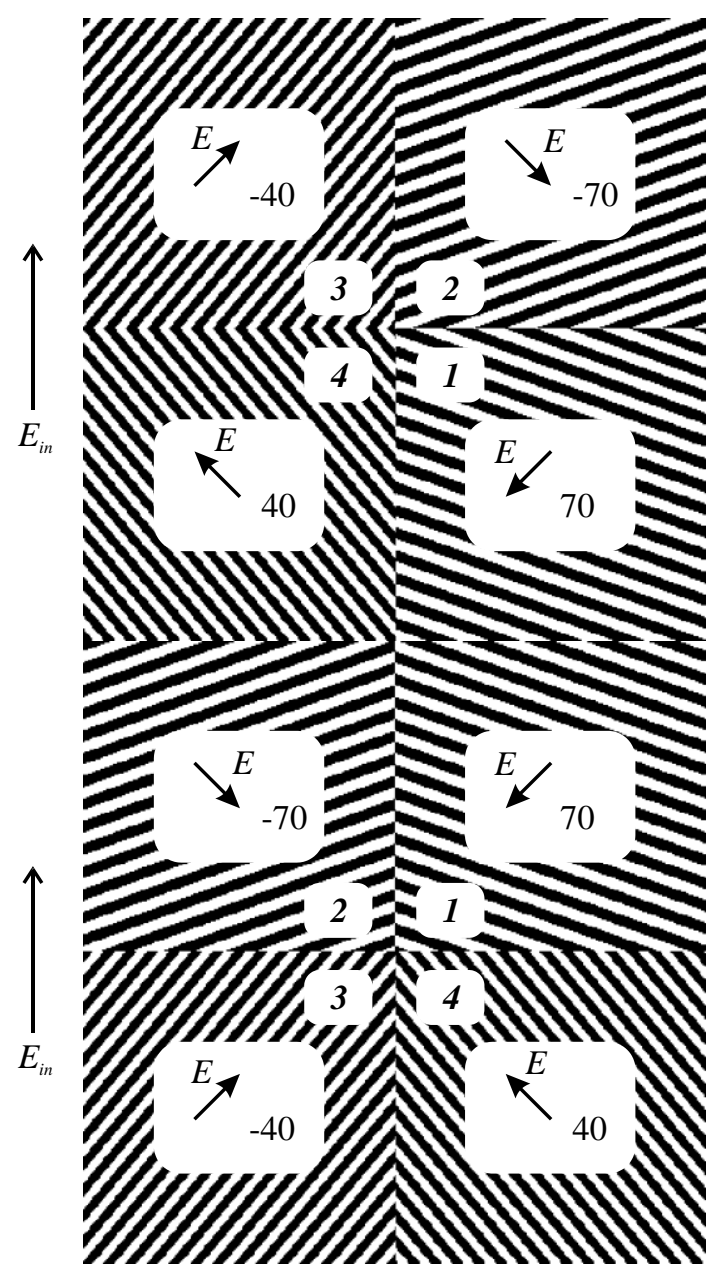

Fig. 1. (a) A SEM image of the central fragment of the fabricated micropolarizer, and the arrangement of gratings of the four-sector polarizer to form (b) azimuthal and (c) radial polarization.

Figure $1 b, c$ depicts the arrangement of gratings in a four-sector micropolarizer to convert linear polarization (vertical arrows) into azimuthal (Fig. $1 b$ ) and radial (Fig. 1c) polarization. The arrows within enumerated squares (1-4) mark the polarization direction of the reflected light. The angles of the grating grooves with the vertical axis are shown in degrees. Figure $1 b, c$ suggests that by merely rotating the polarizer in Fig. 1b by 90 degrees it is not possible to obtain the polarizer in Fig. 1c. To accomplish this requires rearranging the subgratings anticlockwise: 4 to 1,1 to 2,2 to 3 , and 3 to 4 . Also, note that the sub-gratings have different periods.

The performance of the micro-polarizer in Fig. $1 a, b$ was simulated as follows: first, the FDTD-aided simulation of the complex amplitude of the field reflected at the polarizer was implemented using the FullWAVE software. A linearly polarized plane wave of wavelength $532 \mathrm{~nm}$ was assumed to hit the polarizer normally. The mesh of the FDTD method had a $\lambda / 30$ step. The refractive index of the grating grooves and the substrate was $n=0.312+3.17 i$ (gold). The grating feature height was put to be $110 \mathrm{~nm}$, with the substrate thickness being $150 \mathrm{~nm}$. The field distribution at a significant distance from the polarizer was calculated using the Rayleigh-Sommerfeld integral, with the FDTD-aided complex amplitude calculated 200-nm away from the surface taken as an initial field guess. Figure 2 depicts the intensity patterns calculated at distances of $5 \mu \mathrm{m}, 300 \mu \mathrm{m}$, and $500 \mu \mathrm{m}$ from the element, the arrows marking the polarization directions. 


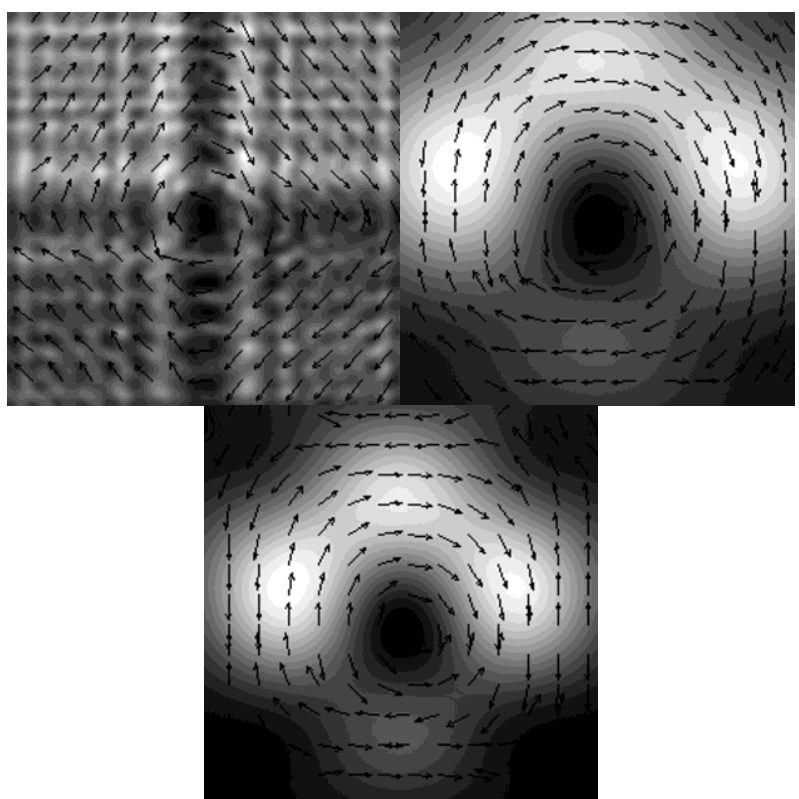

Fig. 2. Intensity patterns and polarization directions for the reflected beam at distances (a) $5.2 \mu \mathrm{m}$, (b) $300.2 \mu \mathrm{m}$, and (c) $500.2 \mu \mathrm{m}$ from the polarizer surface. The image size is (a, b) $20 \times 20 \mu \mathrm{m}$ and (c) $40 \times 40 \mu \mathrm{m}$.

The arrows in Fig. 2 mark the polarization directions. The fact that a number of polarization arrows are oriented in the opposite direction is due to an error of $\pi$ in determining the polarization direction at this point. Following the reflection at the polarizer, the plane wavefront turns into a spherical one at a large distance, whereas the observation remains to be in a plane. As a result, there occurs an extra phase shift that introduces an error of $\pi$ when determining the polarization direction. It is worth noting that such a 'failure' in polarization determination occurs locally, not having an impact on the general pattern of beam polarization.

By choosing a four-sector polarizer, the dependence of the intensity of reflected light on the angle of inclination of the grating grooves can be accounted for. With the angle between the grating's grooves and the incident electric vector $E_{1}$ changing by $\alpha$ degrees, the respective angle between the incident electric vector $E_{1}$ and the reflected electric vector $E_{2}$ changes by $\theta$ degrees. Figure 3 depicts the reflected light intensity $\left|E_{2}\right|^{2}$ and the polarization angle $\theta$ versus the angle $\alpha$.

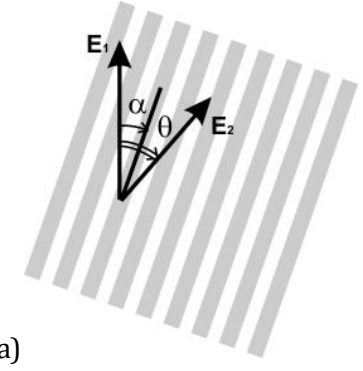

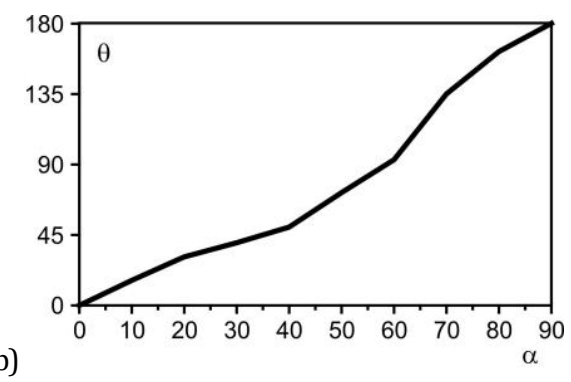

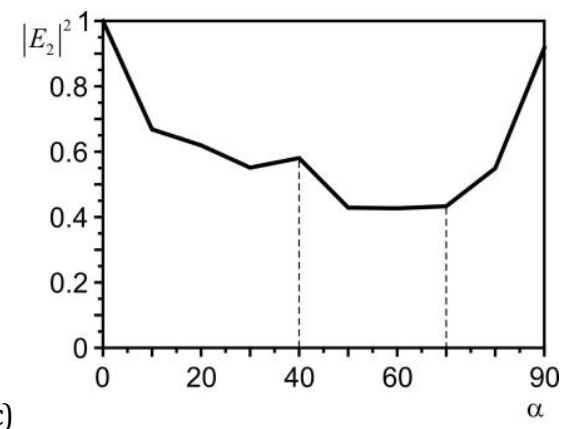

Fig. 3. (a) The angles $\alpha$ and $\theta$ respectively define a change in the groove tilt and the reflected electric field vector, (b) the angle of the polarization vector $\theta$ vs the incident electric vector angle $\alpha$, and (c) reflected field intensity $\left|E_{2}\right|^{2}$ vs the grating's groove angle $\alpha$.

From Fig. 3c, the intensity is seen to vary in a wide range with changing angle of the grating's groove tilt $\alpha$. Hence, we can infer that to be able to form a reflected field with a maximally uniform intensity pattern it will suffice to use just four sectors with differently oriented grating grooves. This enables us to avoid using regions characterized by a maximum reflected intensity (amounting to, or more than 0.7 relative units in Fig 3c). With a four-sector polarizer, the difference between the angles $\theta$ in each adjacent sector needs to be $90^{\circ}$. Taking this and Fig. 3b,c into account, we infer that the angles $\alpha= \pm 40^{\circ}$ and $\pm 70^{\circ}$ are most suitable, as in this case the difference between the reflected intensities from the different sectors is minimal. Then, the respective angles of the polarization rotation are given by $-135^{\circ},-45^{\circ}$, $45^{\circ}$, and $135^{\circ}$. In the course of modeling, the period of the diffraction gratings in all four sectors in Fig. 3 was assumed to be $0.4 \mu \mathrm{m}$ for all angles $\alpha$. Then, to obtain a maximally uniform intensity of the light reflected at four micropolarizer sectors with the angles $\alpha= \pm 40^{\circ}$ and $\pm 70^{\circ}$ the grating periods were optimized. Optimal periods were found to be different, being equal to $0.4 \mu \mathrm{m}$ for the sectors with the angles $\alpha= \pm 40^{\circ}$ and to $0.46 \mu \mathrm{m}$ for the sectors with the angles $\alpha= \pm 70^{\circ}$. Prior to optimizing the grating periods, the ratio of the reflected intensity to the incident one for $\alpha= \pm 40^{\circ}$ and $\alpha= \pm 70^{\circ}$ was, respectively, $58 \%$ and $43.3 \%$ (Fig. 3c). Following the optimization, the reflection efficiency for $\alpha=$ $\pm 70^{\circ}$ reached $51 \%$.

It can also be inferred from Fig. $3 b$ that the nonlinear relationship between the angles $\theta$ and $\alpha$ makes it impossible to synthesize an element that would be able to form both azimuthal and radial polarizations by changing the incident beam polarization plane. This nonlinear relationship follows from the specified range of variation of the grating periods. The grating period is supposed to be smaller than the incident wavelength but not too small, allowing the micropolarizer to be fabricated with acceptable quality by e-beam lithography.

Fabrication of a micropolarizer and generation of azimuthal polarization 
The micropolarizer in Fig. $1 a$ was fabricated by electron beam lithography. A golden layer of thickness 160-180 nm was coated onto a glass substrate, followed by applying a resist layer, on which a foursector grating polarizer pattern was generated by an electron beam lithography (at $30 \mathrm{kV}$ ). Then, the sample was developed by etching with xylene, which dissolved the fragments exposed to the electron beam. Then, using reactive ion etching, the grating-polarizer pattern was transferred into the golden layer, which was etched in places with no resist. Using argon plasma, gold particles were spattered from areas unprotected with the resist. At the final stage, the resist residue was eliminated using oxygen plasma, producing a micropolarizer template 'engraved' in gold. The reactive ion etching time was optimized so as to achieve an etch depth of about $110 \mathrm{~nm}$.

The performance of the fabricated micropolarizer was experimentally tested using a linearly polarized beam of 1-mm width from a 532-nm laser. The beam was focused with a $10 \mathrm{x}$ lens $\mathrm{O}_{1}$ onto the substrate containing the grating micropolarizer (Fig. 4). The size of the spot focused on the micropolarizer was controlled by varying the distance from the lens $\mathrm{O}_{1}$ to the micropolarizer surface. Although in this case, the micropolarizer was not found in the beam waist and the incident wave was spherical, the experimental results we discuss below corroborate that the micropolarizer still operated in a proper way. This may be explained by the fact that while changing from a plane to a spherical wavefront the beam polarization does not acquire an azimuthal component, only acquiring a longitudinal component along the propagation axis, meaning that the angle between the polarization direction in the polarizer plane and the microrelief grooves remains unchanged. The image of the four-sector grating polarizer was displayed in a CCD-camera using a lens $\mathrm{L}_{1}(f \approx 1.5 \mathrm{~cm}$, NA $=0.01$ ). The polarization of the input beam was determined using a polarizer/analyzer $\mathrm{P}_{2}$.

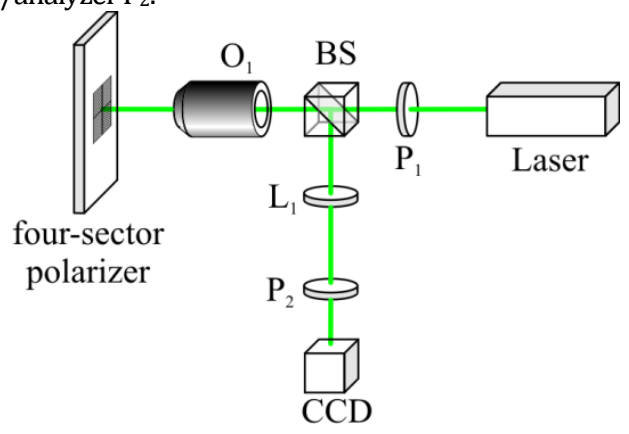

Fig. 4. Experimental setup: $\mathrm{P}_{1}, \mathrm{P}_{2}$ are linear polarizers, $\mathrm{BS}$ is a nonpolarizing light-splitting cube, $\mathrm{O}_{1}$ is a $10 \times$ lens ( $\left.\mathrm{NA}=0.25\right), \mathrm{L}_{1}$ is a lens $(\mathrm{f} \approx 1.5 \mathrm{~cm}$ ), and a CCD-camera.

Figure 5 depicts the central fragment of a four-sector polarization converter illuminated by a linearly polarized Gaussian beam from a 532-nm laser. The illuminated area is somewhat smaller than the entire micropolarizer of size $100 \times 100 \mu \mathrm{m}$. The linear polarization of the light outgoing from the splitting cube and incident on the micropolarizer was checked using a standard polarizer. Following reflection at the micropolarizer, the polarization state of light was changed. Figure 6 depicts images of the micropolarizer in Fig. $1 a$ with a polarizer $\mathrm{P}_{2}$ placed in front of the CCD-camera and making angles $0^{\circ}$ (a), $90^{\circ}$ (b), $-45^{\circ}$ (c), and $45^{\circ}$ (d) with the incident light polarization axis. If the numerically simulated electric field intensity (Fig. 2a) is multiplied by the Jones matrix of a polarizer rotated by the angle $45^{\circ}$ (or $-45^{\circ}$ ), it can numerically be shown in which way the intensity pattern in Fig. 2a is converted following the passage through the polarizer (Fig. 1a). Figures $6 \mathrm{e}$ and $6 \mathrm{f}$ depict the simulated intensity patterns after the incident beam was reflected at the four-sector polarizer and then transmitted through the analyzer P2, with the analyzer in Figs. $6 \mathrm{e}$ and $6 \mathrm{f}$ being, respectively, rotated by $-45^{\circ}$ and $45^{\circ}$ about the incident light polarization axis. The simulated intensity pattern is square-shaped because the incident wave was assumed to be plane, whereas the experiment was conducted using a Gaussian beam. However, despite the incident beams being different, the experimental patterns in Figs. $6 \mathrm{c}$ and $6 \mathrm{~d}$ are seen to be in good agreement with the simulated patterns in Figs. 6e and $6 \mathrm{f}$.

Figure 7 depicts the far-field intensity pattern for a beam reflected at the micropolarizer in Fig. $1 a$, which occurs in the focal plane of lens $\mathrm{L}_{1}$. The CCD-camera image was obtained using a $20 \times$ lens.

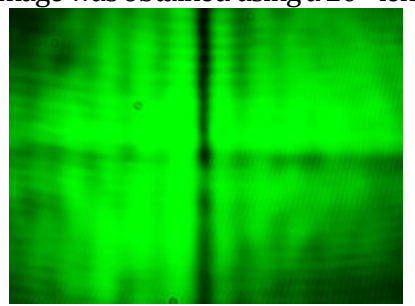

Fig. 5. Image of the micropolarizer in Fig. 4 obtained in laser light without an output polarizer.
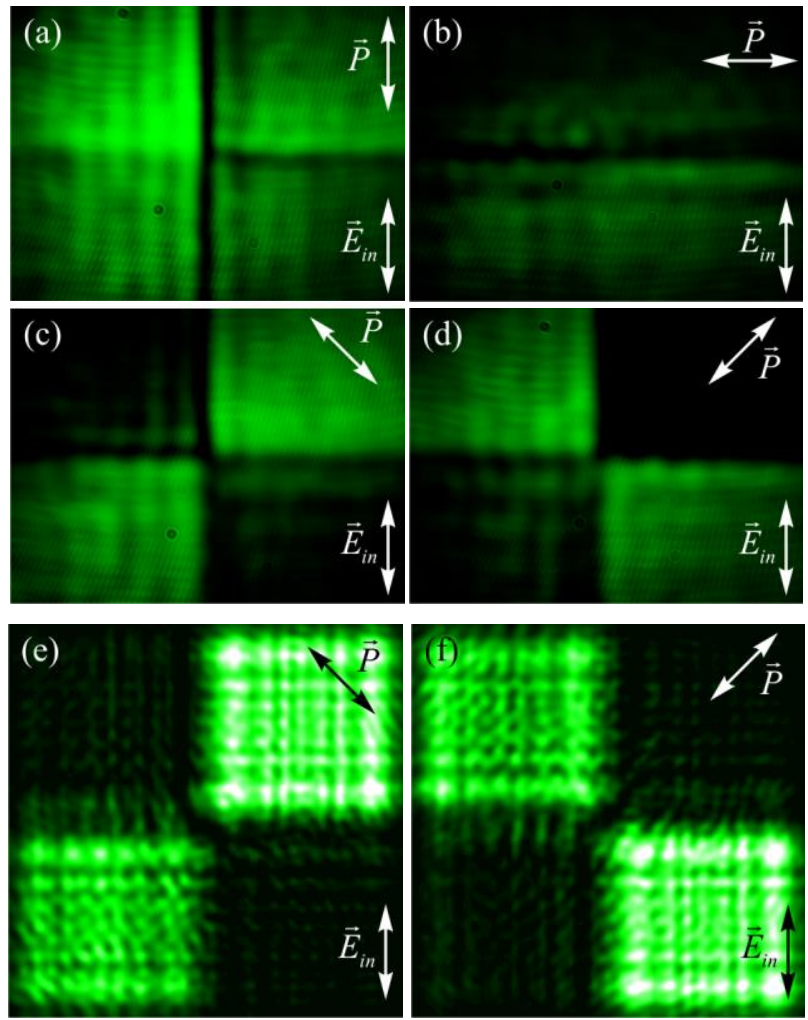

Fig. 6. Image of the micropolarizer in Fig. 4 in the laser light. In front of the camera was an output polarizer/analyzer $\mathrm{P}_{2}$, making an angle of $0^{\circ}$ (a), $90^{\circ}(\mathrm{b}),-45^{\circ}$ (c), and $45^{\circ}$ (d) with the incident beam. The frame size was $4 \mathrm{~mm} \times 3 \mathrm{~mm}$. Numerically simulated intensity patterns of the beam in Fig. $2 \mathrm{a}$, which was reflected at the four-sector polarizer and then transmitted through the analyzer P2, with the analyzer in Figs. $6 \mathrm{e}$ and $6 \mathrm{f}$ being, respectively, rotated by $-45^{\circ}$ and $45^{\circ}$ with respect to the incident light polarization axis. 


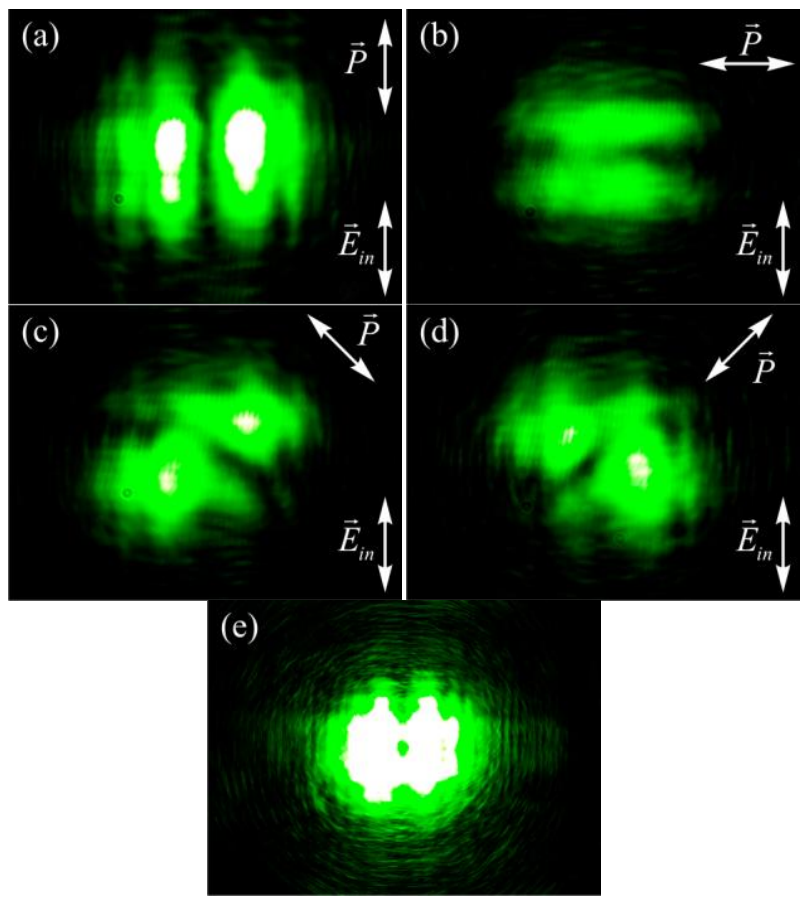

Fig. 7. Far-field diffraction patterns for a laser beam reflected at the four-sector micropolarizer. In front of the camera was an output polarizer/analyzer $\mathrm{P}_{2}$ making the angles of $0^{\circ}(\mathrm{a}), 90^{\circ}(\mathrm{b}),-45^{\circ}(\mathrm{c})$, and $45^{\circ}$ (d) with the incident beam polarization axis. The annular intensity with no polarizer (e). The frame size is $4 \mathrm{~mm} \times 3 \mathrm{~mm}$.

Based on Fig. 7, we can infer that the four-sector micropolarizer in Fig. $1 a$ converts an incident linearly polarized beam into an azimuthally polarized light beam. However, the intensity of the reflected beam is not distributed uniformly over the ring. The reason is that the four-sector polarizer in Fig. 1a is devoid of radial symmetry. It should be noted that a classical work on the sharp focus of radially polarized laser light [17] also utilized a four-sector polarizer, which was made up of mutually rotated half-wave plates. Hence, we can infer that four sectors would suffice to form a radially [17] or azimuthally polarized beam. Note that the use of a four-sector micropolarizer in our previous work [8] made it possible to focus the laser beam into a focal spot of area $(0.35 \times 0.38) \lambda$, meanwhile using the four-sector polarizer made up of half-wave plates [17] enabled a focal spot of size $0.45 \lambda$.

\section{Optical vortices with different polarization of the initial beam}

In this Section, using the Rayleigh-Sommerfeld integrals we derive conditions for an on-axis intensity maximum for optical vortices with different initial polarization. The propagation of coherent monochromatic light in free space is known to be described by the vector Rayleigh-Sommerfeld integrals [18]:

$$
\begin{aligned}
& E_{x, y}(\rho, \theta, z)=-\frac{1}{2 \pi} \int_{0}^{\infty} \int_{0}^{2 \pi} E_{x, y}(r, \varphi, 0) \times \\
& \times \frac{\partial}{\partial z}\left(\frac{e^{i k R}}{R}\right) r d r d \varphi,
\end{aligned}
$$

$$
\begin{aligned}
& E_{z}(\rho, \theta, z)=\frac{1}{2 \pi} \int_{0}^{\infty} \int_{0}^{2 \pi}\left[E_{x}(r, \varphi, 0) \frac{\partial}{\partial x}\left(\frac{e^{i k R}}{R}\right)+\right. \\
& \left.+E_{y}(r, \varphi, 0) \frac{\partial}{\partial y}\left(\frac{e^{i k R}}{R}\right)\right] r d r d \varphi,
\end{aligned}
$$

where

$$
\begin{aligned}
& R=\left(z^{2}+r^{2}+\rho^{2}-2 r \rho \cos (\varphi-\theta)\right)^{1 / 2} \approx \\
& \approx z+\frac{r^{2}}{2 z}+\frac{\rho^{2}}{2 z}-\frac{r \rho}{z} \cos (\varphi-\theta),
\end{aligned}
$$

$k$ is the wavenumber, $(\rho, \theta, z)$ are the cylindrical coordinates, and $(r, \varphi)$ are the polar coordinates in the initial plane $z=0$. The approximate relation in (3) is valid in the paraxial case, when $z>>r, \rho$.

Let the initial light field be in the form of a radially polarized optical vortex with the complex amplitude:

$$
\left\{\begin{array}{l}
E_{r}(r, \varphi, 0)=A_{r}(r) e^{i n \varphi}, \\
E_{\varphi}(r, \varphi, 0)=0 .
\end{array}\right.
$$

Then, integrals (1)-(3) in the paraxial approximation are given by

$$
\begin{aligned}
& E_{r}(\rho, \theta, z)=\frac{(-i)^{n} k}{2 z} \exp \left(i k z+i n \theta+\frac{i k \rho^{2}}{2 z}\right) \\
& \int_{0}^{\infty} A_{r}(r) e^{\frac{i k r^{2}}{2 z}}\left[J_{n-1}\left(\frac{k r \rho}{z}\right)-J_{n+1}\left(\frac{k r \rho}{z}\right)\right] r d r, \\
& E_{\varphi}(\rho, \theta, z)=\frac{(-i)^{n-1} k}{2 z} \exp \left(i k z+i n \theta+\frac{i k \rho^{2}}{2 z}\right) \\
& \int_{0}^{\infty} A_{r}(r) e^{\frac{i k r^{2}}{2 z}}\left[J_{n-1}\left(\frac{k r \rho}{z}\right)+J_{n+1}\left(\frac{k r \rho}{z}\right)\right] r d r, \\
& E_{z}(\rho, \theta, z)=\frac{(-i)^{n-1} k}{2 z^{2}} \exp \left(i k z+i n \theta+\frac{i k \rho^{2}}{2 z}\right) \\
& \int_{0}^{\infty} A_{r}(r) e^{\frac{i k k^{2}}{2 z}}\left\{2 r J_{n}\left(\frac{k r \rho}{z}\right)+\right. \\
& \left.i \rho\left[J_{n+1}\left(\frac{k r \rho}{z}\right)-J_{n-1}\left(\frac{k r \rho}{z}\right)\right]\right\} r d r .
\end{aligned}
$$

From (5)-(7) we can infer that the initial radial polarization of a vortex field does not remain unchanged during propagation, turning into a mixture of radially and azimuthally polarized components. Equations (5) and (6) also suggest that taking the initial radially polarized non-vortex beam $(n=0)$ and considering that $J_{-n}(x)=(-1)^{n} J_{n}(x)$, we obtain the azimuthal $E$-field component with zero amplitude: $E_{\varphi}(\rho=0, \theta, z)=0$. Thus, the radially polarized and radially symmetric light field $E_{r}(r, \varphi, 0)=A_{r}(r)$ retains its radial polarization during propagation.

Note that at $n>1$ and $n<-1$, all $E$-field components in (5)-(7) equal zero on the optical axis $(\rho=0)$ ). If $n=0$, only longitudinal components take non-zero values on the optical axis $(\rho=0): E_{z}(\rho=0, \theta, z) \neq 0$, whereas at $n= \pm 1$, only transverse $E$-field components are non-zero on the optical axis: $E_{r}(\rho=0, \theta, z)=\mp i E_{\varphi}(\rho=0, \theta, z) \neq 0$. Thus, there are two ways to form an on-axis focal spot: 1 ) by means of a radially 
polarized non-vortex ( $n=0)$ initial field and 2) using an initial radially polarized vortex field at $n= \pm 1$. The latter approach is preferable because the annular on-axis focal spot is contributed to by the transverse $E$-field components. The transverse $E$-field components propagate along the optical axis and can be used by the observer. With the former approach, when the focal spot is obtained using a nonvortex radially symmetric field, light does not propagate along the optical axis.

Let the initial field be defined by an azimuthally polarized optical vortex with a complex amplitude:

$$
\left\{\begin{array}{l}
E_{\varphi}(r, \varphi, 0)=A_{\varphi}(r) e^{i n \varphi}, \\
E_{r}(r, \varphi, 0)=0 .
\end{array}\right.
$$

Then, integrals (1)-(3) in the paraxial approximation take the form:

$$
\begin{gathered}
E_{r}(\rho, \theta, z)=\frac{(-i)^{n+1} k}{2 z} \exp \left(i k z+i n \theta+\frac{i k \rho^{2}}{2 z}\right) \\
\int_{0}^{\infty} A_{\varphi}(r) e^{\frac{i k r^{2}}{2 z}}\left[J_{n-1}\left(\frac{k r \rho}{z}\right)+J_{n+1}\left(\frac{k r \rho}{z}\right)\right] r d r, \\
E_{\varphi}(\rho, \theta, z)=\frac{(-i)^{n} k}{2 z} \exp \left(i k z+i n \theta+\frac{i k \rho^{2}}{2 z}\right) \\
\int_{0}^{\infty} A_{\varphi}(r) e^{\frac{i k r^{2}}{2 z}}\left[J_{n-1}\left(\frac{k r \rho}{z}\right)-J_{n+1}\left(\frac{k r \rho}{z}\right)\right] r d r, \\
E_{z}(\rho, \theta, z)=\frac{(-i)^{n+1} k \rho}{2 z^{2}} \exp \left(i k z+i n \theta+\frac{i k \rho^{2}}{2 z}\right) \\
\int_{0}^{\infty} A_{\varphi}(r) e^{\frac{i k r^{2}}{2 z}}\left[J_{n-1}\left(\frac{k r \rho}{z}\right)+J_{n+1}\left(\frac{k r \rho}{z}\right)\right] r d r,
\end{gathered}
$$

From comparison of (9) and (11), the radial and longitudinal components of the $E$-field are seen to be related as

$$
E_{z}(\rho, \theta, z)=\frac{\rho}{z} E_{r}(\rho, \theta, z) .
$$

From (9)-(11) it follows that 1) the initial field defined by an azimuthally polarized vortex does not retain azimuthal polarization during propagation, becoming a mixture of radial and azimuthal polarization. From (9) and (11) it also follows that given a non-vortex azimuthally polarized initial field $(n=0)$ and considering that $J_{-n}(x)=(-1)^{n} J_{n}(x)$, the amplitude of the radial component of the $E$ field is zero: $E_{r}(\rho=0, \theta, z)=0$. Hence, an azimuthally polarized and radially symmetric field $E_{\varphi}(r, \varphi, 0)=A_{\varphi}(r)$ remains azimuthally polarized during propagation. It can be noted that the above conclusions relating to the field in (9)-(11) coincide with the conclusions relating to the field in (5)-(7), with the term 'azimuthal' being replaced with 'radial'. However, the subsequent conclusions are going to be different. At $n \neq \pm 1$, all $E$-field components in (9)-(11) are zero on the optical axis $(\rho=0)$. The longitudinal component (12) always equals zero on the optical axis: $E_{z}(\rho=0, \theta, z)=0$. If $n= \pm 1$, only transverse E-field components take non-zero values on the optical axis: $E_{r}(\rho=0, \theta, z)=\mp i E_{\varphi}(\rho=0, \theta, z) \neq 0$. Thus, using the initial azimuthally polarized vortex field with the topological charge $n= \pm 1$, the on-axis focal spot can be generated in a unique way. The other conditions being the same, the resulting circular focal spot should be of the same size as for the radially polarized initial vortex field with \pm 1 . This can be inferred from the fact that (5) and (6) are functionally equivalent to (9) and (11). Similar results have been obtained previously for a particular amplitude function $A(r)$ [19].

\section{A spiral phase plate in an azimuthally polarized laser beam}

In the experimental setup in Fig. 8, a linearly polarized beam from a 532-nm laser was focused using a $10 \mathrm{x}$ objective $\mathrm{O}_{1}$ onto a substrate coated with a four-zone grating polarizer (Fig. 1a). Following the reflection at the micropolarizer, the beam passed through a spiral phase plate (SPP) with $n=1$ and lens $L_{1}(f \approx 1.5 \mathrm{~cm})$, forming a focal spot, with the intensity distribution observed using a $20 \mathrm{x}$ objective $\mathrm{O}_{2}$.

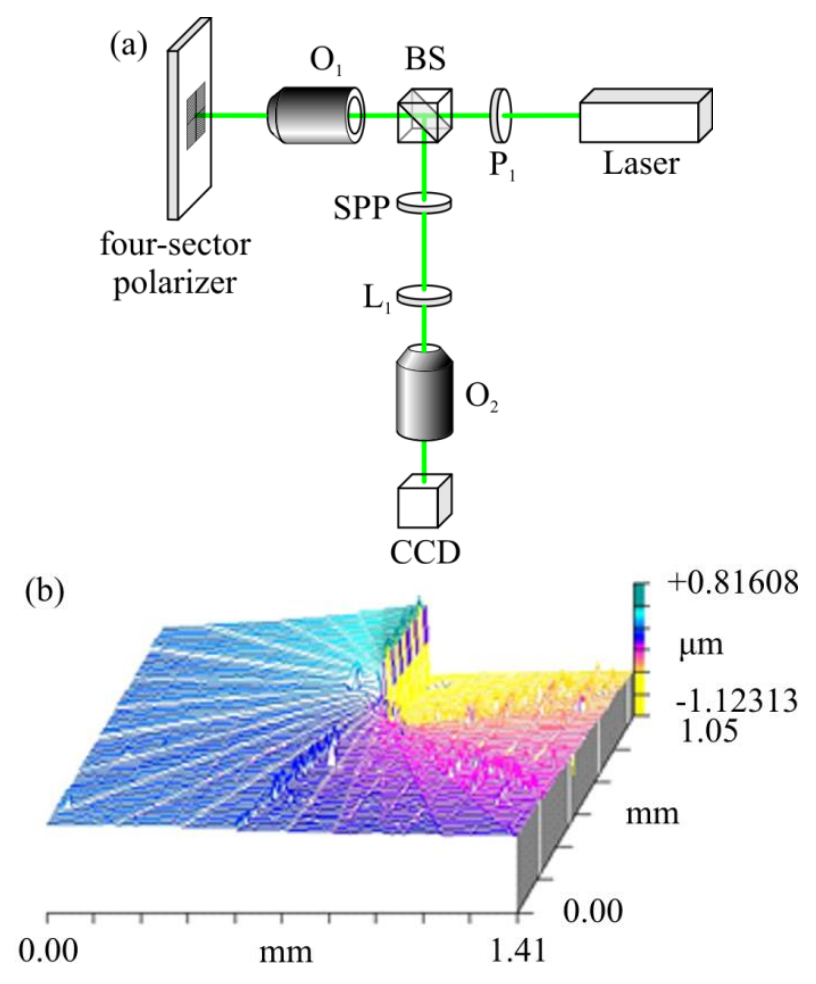

Fig. 8. (a) Experimental setup: $\mathrm{P}_{1}$ is a linear polarizer, $\mathrm{O}_{1}$ is a $10 \times$ objective (NA $=0.25), \mathrm{L}_{1}$ is a lens $(\mathrm{f} \approx 1.5 \mathrm{~cm}), \mathrm{O}_{2}$ is a $20 \times$ objective $(\mathrm{NA}=0.4), \mathrm{CCD}$ is a CCD-camera, and SPP is a spiral phase plate with $\mathrm{n}=1$ (b).

A 32-level SPP (Fig.8b) was fabricated on a low-contrast negative $X$ AR-N Z220 e-beam resist with variable-dose patterning [20]. The SPP size is $2.5 \mathrm{~mm} \times 2.5 \mathrm{~mm}$ and the pixel size is $5 \mu \mathrm{m} \times 5 \mu \mathrm{m}$. The microrelief depth is $1075 \mathrm{~nm}(\lambda=532 \mathrm{~nm})$.

Figure 9 depicts the intensity pattern in the focus without (Fig. 9a) and with (Fig. 9b) a spiral phase plate put in the beam's path.

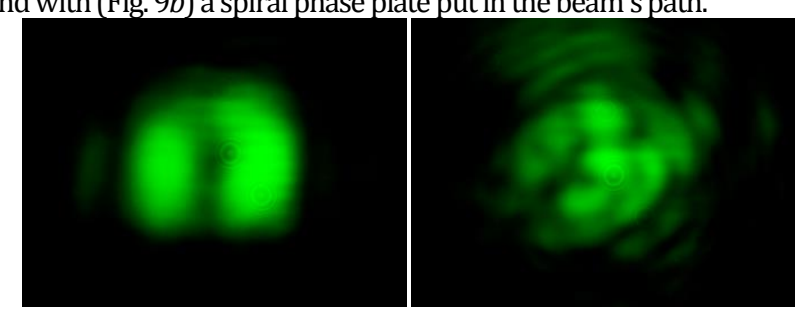


Fig. 9. Lens-aided focal spots from an azimuthally polarized beam (a) without and (b) with a SPP with $n=1$ in the beam's path. Frame size is $(2.7 \times 2) \mathrm{mm}$.
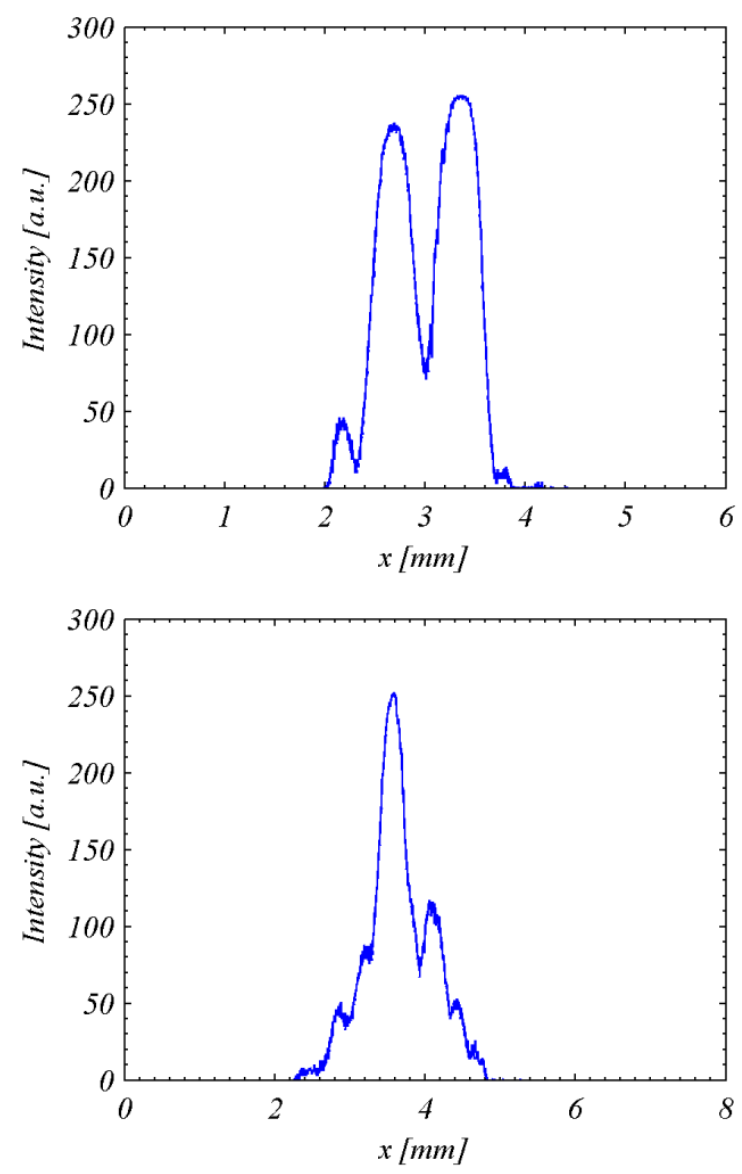

Fig. 10. Lens-focused intensity profiles of Fig. 8 (a) without and (b) with a SPP with $\mathrm{n}=1$ in the beam's path.

From Fig. 9 the azimuthally polarized beam is seen to be focused by the lens into a near-annular focal spot. It is also seen that by introducing a SPP into the beam's path the focal intensity pattern is changed to a central intensity peak with side lobes. This can clearly be seen from Fig. 10, which shows profiles of the intensity patterns in Fig. 9.

To verify the experimental results, we additionally simulated the focusing of an azimuthally polarized beam with $n=1$ using the Richards-Wolf formulae. In the first run of simulation, the lens $(\mathrm{NA}=0.3$ ) was assumed to focus a plane wave in which polarization changed sector-wise, depending on the value of the azimuthal angle $\varphi$. In the second run of simulation, the plane wave from the first run was complemented with an optical vortex. The resulting intensity patterns are shown in Fig. 11.

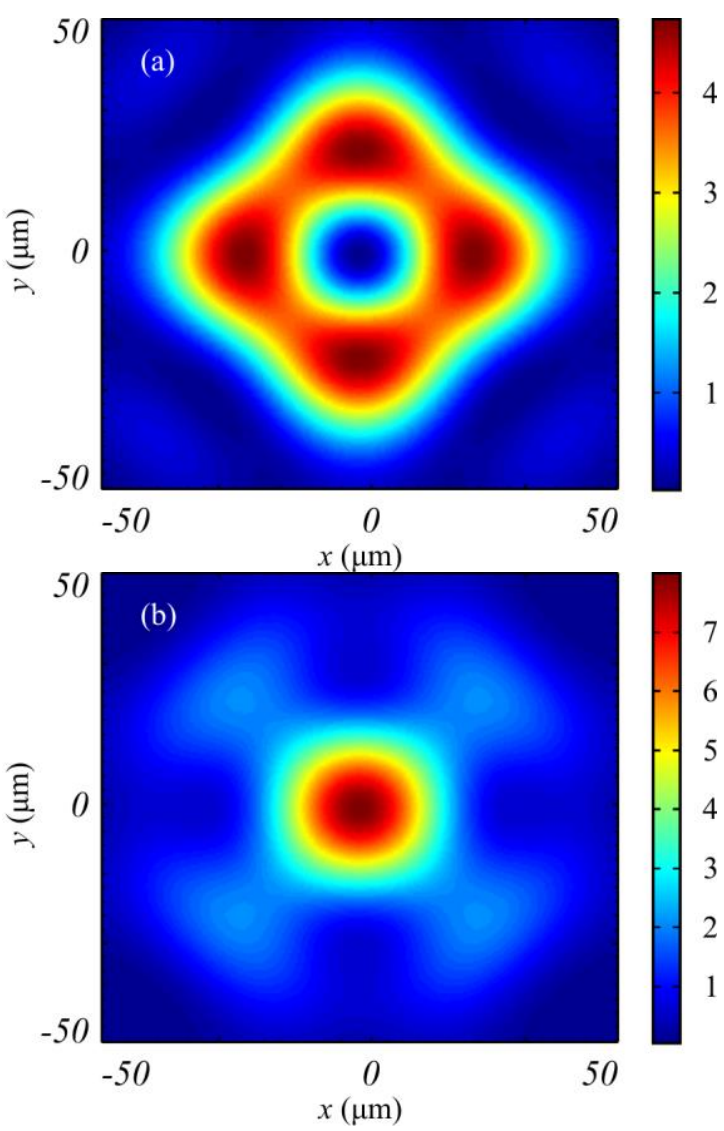

Fig. 11. Intensity patterns in the lens focus for an azimuthally polarized beam (a) without and (b) with a SPP put in the beam's path.

The simulation results in Fig. 11 show that the focal spot size (b) coincides with the size of a low-intensity region in the center of the four-sector ring (a). A similar pattern can be seen in the experiment in Figs. 9 and 10. From Fig. 11, the shape of the intensity pattern is seen to change, with the annular pattern changing to the central intensity peak with side-lobes. This is in agreement with the experimental patterns in Fig. 9, in which there may be seen a nonuniform ring obtained without a SPP (Fig. 9a) and a central intensity peak with sidelobes obtained using a SPP (Fig. 9b). Note that patterns in Fig. 9b and Fig. $11 \mathrm{~b}$ are rotated relative to each other by 45 degrees. It is also worth noting that the dark region in the annular intensity distribution in Fig. $11 \mathrm{~b}$ is approximately equal in size to the central peak in Fig. 11a. Thus, the experiment is in agreement with the calculations. The distance between two intensity peaks in Fig. 10a is $0.66 \mathrm{~mm}$, whereas the full size of the central peak in Fig. 10b, which is defined as the distance between two minima on the both sides of the central peak, is $0.67 \mathrm{~mm}$

\section{Conclusions}

Summing up, we have for the first time designed and manufactured a four-sector binary subwavelength reflecting micropolarizer in a gold film to convert a linearly polarized light beam into an azimuthally polarized beam. It should be noted that the four-sector polarizer composed of subwavelength gratings to convert linear polarization into the radial one [7] is different from the four-sector micropolarizer in Fig. 1 to convert linear polarization into the azimuthal one. Using FDTD-aided simulation and physical experiments, we have shown the manufactured four-sector micropolarizer to form an azimuthally polarized beam in the near- and far-field diffraction regions. Using 
vector Rayleigh-Sommerfeld integrals, conditions for the on-axis intensity peak have been deduced for circularly, azimuthally, and radially polarized vortices. It has been numerically and experimentally shown that by introducing a SPP with $n=1$ into an azimuthally polarized beam the focal intensity pattern can be changed from 'annular' to a central intensity peak for low numerical apertures $(\mathrm{NA}<0.4)$.

\section{Acknowledgements}

Funding Information. RF President's grant for support of young candidates of science (MK-9019.2016.2); Russian Foundation for Basic Research (RFBR) (14-07-97039, 14-29-07133, 15-07-01174, 15-3720723; 16-07-00990).

\section{References}

1. V. V. Kotlyar, O. K. Zalyalov, "Design of diffractive optical elements modulating polarization," Optik 103, 125-130 (1996).

2. Z. Bomzon, V. Kleiner, and E. Hasman, "Pancharatnam-Berry phase in space-variant polarization-state manipulations with subwavelength gratings," Opt. Lett. 26, 1424-1426 (2001)

3. Z. Bomzon, G. Biener, V. Kleiner, and E. Hasman, "Radially and azimuthally polarized beams generated by space-variant dielectric subwavelength gratings," Opt. Lett. 27, 285-287 (2002)

4. G. M. Lerman and U. Levy, "Generation of a radially polarized light beam using space-variant subwavelength gratings at $1064 \mathrm{~nm}$," Opt. Lett. 33, 2782-2784 (2008)

5. T. Kaempfe, P. Sixt, D. Renaud, A. Lagrange, F. Perrin, and O. Parriaux, "Segmented subwavelength silicon gratings manufactured by high productivity microelectronic technologies for linear to radial/azimuthal polarization conversion," Optical Engineering 53, 107105 (2014).

6. Z. Ghadyani, I. Vartiainen, I. Harder, W. Iff, A. Berger, N. Lindlein, and $\mathrm{M}$. Kuittinen, "Concentric ring metal grating for generating radially polarized light," Appl. Opt. 50, 2451-2457 (2011)

7. A. G. Nalimov, L. O'Faolain, S. S. Stafeev, M. I. Shanina, and V. V. Kotlyar, "Reflected four-zones subwavelenghth mictooptics element for polarization conversion from linear to radial," Computer Optics 38, 229-236 (2014).

8. S. S. Stafeev, L. O'Faolain, V. V. Kotlyar, and A. G. Nalimov, "Tight focus of light using micropolarizer and microlens," Appl. Opt. 54, 4388-4394 (2015)

9. L.E. Helseth, "Optical vortices in focal regions," Optics Communications 229, 85-91 (2004).

10. Z. Zhang, J. Pu, and X. Wang, "Tight focusing of radially and azimuthally polarized vortex beams through a uniaxial birefringent crystal," Appl. Opt. 47, 1963-1967 (2008)

11. V. V. Kotlyar and A. A. Kovalev, "Nonparaxial propagation of a Gaussian optical vortex with initial radial polarization," J. Opt. Soc. Am. A 27, 372-380 (2010)

12. X. Hao, C. Kuang, T. Wang, and X. Liu, "Phase encoding for sharper focus of the azimuthally polarized beam," Opt. Lett. 35, 3928-3930 (2010)

13. S. Wang, X. Li, J. Zhou, and M. Gu, "Ultralong pure longitudinal magnetization needle induced by annular vortex binary optics," Opt. Lett. 39, 5022-5025 (2014)

14. Z. Nie, W. Ding, D. Li, X. Zhang, Y. Wang, and Y. Song, "Spherical and sub-wavelength longitudinal magnetization generated by $4 \pi$ tightly focusing radially polarized vortex beams," Opt. Express 23, 690-701 (2015)

15. Z. Chen and D. Zhao, "4Pi focusing of spatially modulated radially polarized vortex beams," Opt. Lett. 37, 1286-1288 (2012)

16. X. Li, P. Venugopalan, H. Ren, M. Hong, and M. Gu, "Super-resolved pure-transverse focal fields with an enhanced energy density through focus of an azimuthally polarized first-order vortex beam," Opt. Lett. 39, 5961-5964 (2014)

17. R. Dorn, S. Quabis, and G. Leuchs, "Sharper focus for a radially polarized light beam," Physical Review Letters 91, 233901 (2003).

18. J. A. C. Veerman, J. J. Rusch, and H. P. Urbach, "Calculation of the Rayleigh-Sommerfeld diffraction integral by exact integration of the fast oscillating factor," J. Opt. Soc. Am. A 22, 636-646 (2005).

19. V.V. Kotlyar, A.A. Kovalev, "Nonparaxial propagation of a Gaussian optical vortex with initial radial polarization", J. Opt. Soc. Am. A 27, 372-380 (2010).

20. V.V. Kotlyar, A.A. Almazov, S.N. Khonina, V.A. Soifer, H. Elfstrom, J. Turunen, "Generation of phase singularity through diffracting a plane or Gaussian beam by a spiral phase plate", J. Opt. Soc. Am. A 22, 849-861 (2005). 